Gut and Liver, Vol. 12, No. 4, July 2018, pp. 463-470

\title{
Prospective Comparison of Intraductal Ultrasonography-Guided Transpapillary Biopsy and Conventional Biopsy on Fluoroscopy in Suspected Malignant Biliary Strictures
}

\author{
Hyun Su Kim ${ }^{1}$, Jong Ho Moon ${ }^{1}$, Yun Nah Lee ${ }^{1}$, Hyun Jong Choi ${ }^{1}$, Hyun Woo Lee ${ }^{1}$, Hee Kyung Kim² ${ }^{2}$ Tae Hoon Lee ${ }^{1}$, \\ Moon Han $\mathrm{Choi}^{1}$, Sang-Woo Cha ${ }^{1}$, Young Deok Cho ${ }^{1}$, and Sang-Heum Park ${ }^{1}$ \\ ${ }^{1}$ Digestive Disease Center and Research Institute, Department of Internal Medicine, Soonchunhyang University School of Medicine, and \\ ${ }^{2}$ Department of Pathology, Soonchunhyang University School of Medicine, Bucheon, Korea
}

Background/Aims: In suspected malignant biliary strictures (MBSs), the diagnostic yield of endoscopic retrograde cholangiopancreatography (ERCP)-based tissue sampling is limited. Transpapillary forceps biopsy (TPB) under intraductal ultrasonography (IDUS) guidance is expected to improve the diagnostic accuracy in patients with indeterminate biliary strictures. We evaluated the usefulness of IDUS-guided TPB in patients with suspected MBS. Methods: Consecutive patients with suspected MBS were prospectively enrolled in the study. ERCP with IDUS was performed in all patients. Both conventional TPB and IDUS-guided TPB on fluoroscopy were performed in each patient. The primary outcome was the diagnostic accuracy of conventional TPB and IDUS-guided TPB. Results: The technical success rate of IDUS-guided TPB was $97.0 \%$ (65/67 patients). Of these 65 patients, the final diagnosis was malignancy in 61 patients (93.8\%). On IDUS, the most common finding of IDUS was an intraductal infiltrating lesion in 29 patients (47.5\%). The overall diagnostic accuracy was significantly higher using IDUS-guided TPB than that using conventional TPB (90.8\% vs $76.9 \%, p=0.027)$. According to the subgroup analysis based on the tumor morphology, IDUS-guided TPB had a significantly higher cancer detection rate than conventional TPB for intraductal infiltrating lesions (89.6\% vs $65.5 \%, p=0.028$ ). Conclusions: IDUS-guided TPB appears to improve the accuracy of histological diagnosis in patients with MBS. (Gut Liver 2018;12:463-470)

Key Words: Malignant biliary stricture; Intraductal ultrasonography; Cholangiopancreatography, endoscopic retrograde; Biopsy

\section{INTRODUCTION}

Endoscopic retrograde cholangiopancreatography (ERCP) provides radiologic images of bile duct strictures and enables tissue sampling for histopathological diagnosis. Although transpapillary forceps biopsy (TPB) produces the highest diagnostic yield, the sensitivity for diagnosis of malignant biliary stricture (MBS) is affected by several factors and reportedly varies from $48.1 \%$ to $73 \% .^{1-3}$ Conventional TPB on only fluoroscopy guidance is insufficient to precisely target and sample the tumor during TPB. ${ }^{2,3}$

Intraductal ultrasonography (IDUS) has demonstrated high diagnostic sensitivity and specificity in previous studies ${ }^{4,5}$ and has been used along with fluoroscopy during ERCP to differentiate malignant from benign strictures and confirm local $\mathrm{T}$ staging of tumors. IDUS provides real-time, high-resolution images of the bile duct wall and the surrounding structures. ${ }^{5,6}$ Based on these advantages, IDUS guidance during TPB is expected to provide more accurate biopsy results via ultrasonic visualization of the mass, wall layer, and use of biopsy forceps than fluoroscopic guidance. In particular, IDUS-guided TPB allows for opening and closing of the forceps at the location most likely to yield diagnostic tissue on IDUS.

The aim of this study was to evaluate the diagnostic accuracy of IDUS-guided TPB compared with conventional TPB on fluoroscopy in patients with suspected MBS.

\footnotetext{
Correspondence to: Jong Ho Moon

Digestive Disease Center and Research Institute, Department of Internal Medicine, Soonchunhyang University Bucheon Hospital, 170 Jomaru-ro, Wonmi-gu, Bucheon 14584, Korea

Tel: +82-32-621-5094, Fax: +82-32-621-5080, E-mail: jhmoon@schmc.ac.kr

Received on May 8, 2017. Revised on July 27, 2017. Accepted on September 29, 2017. Published online February 8, 2018 pISSN 1976-2283 eISSN 2005-1212 https://doi.org/10.5009/gnl17205

(a) This is an Open Access article distributed under the terms of the Creative Commons Attribution Non-Commercial License (http://creativecommons.org/licenses/by-nc/4.0) which permits unrestricted non-commercial use, distribution, and reproduction in any medium, provided the original work is properly cited.
} 


\section{MATERIALS AND METHODS}

\section{Patients}

Consecutive patients diagnosed with suspected extrahepatic MBS and showing obstructive jaundice were prospectively enrolled. The inclusion criteria were: (1) clinical presentation of obstructive jaundice consistent with a biliary stricture; (2) suspected MBS on imaging studies, including computed tomography and/or magnetic resonance cholangiopancreatography; (3) age 18 years or older; and (4) ability to provide informed con-
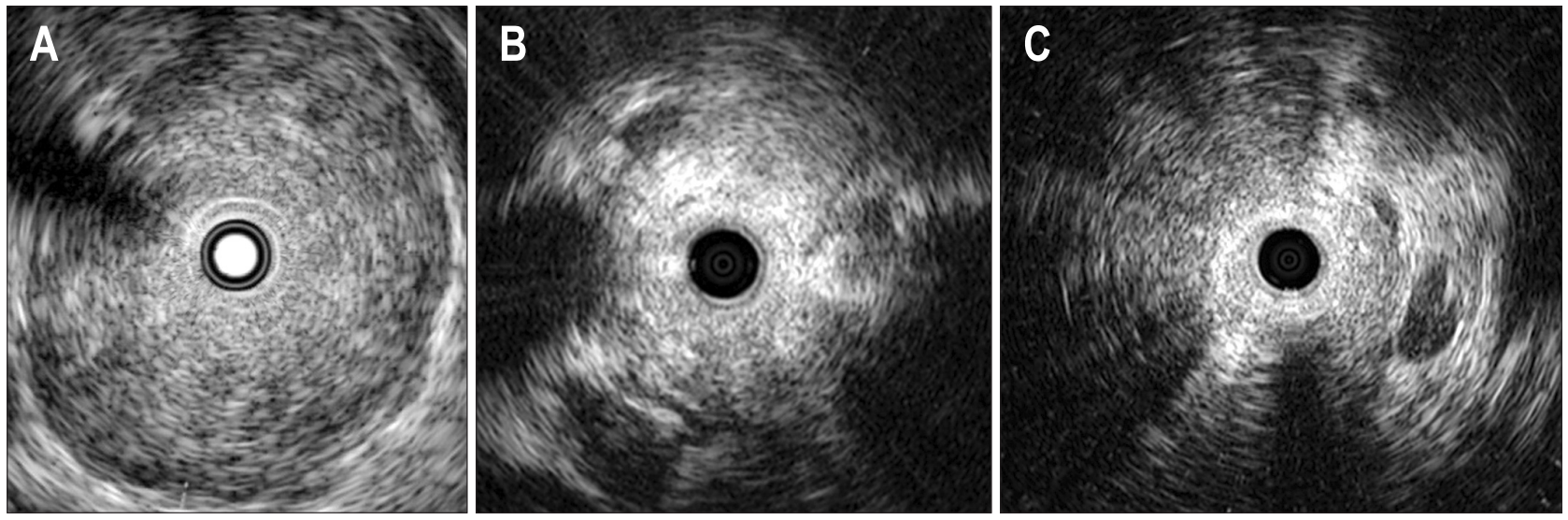

Fig. 1. Classification of malignant biliary strictures by morphology on intraductal ultrasonography. (A) Nodular lesion, (B) intraductal infiltrating lesion, and (C) extrinsic compressed lesion.
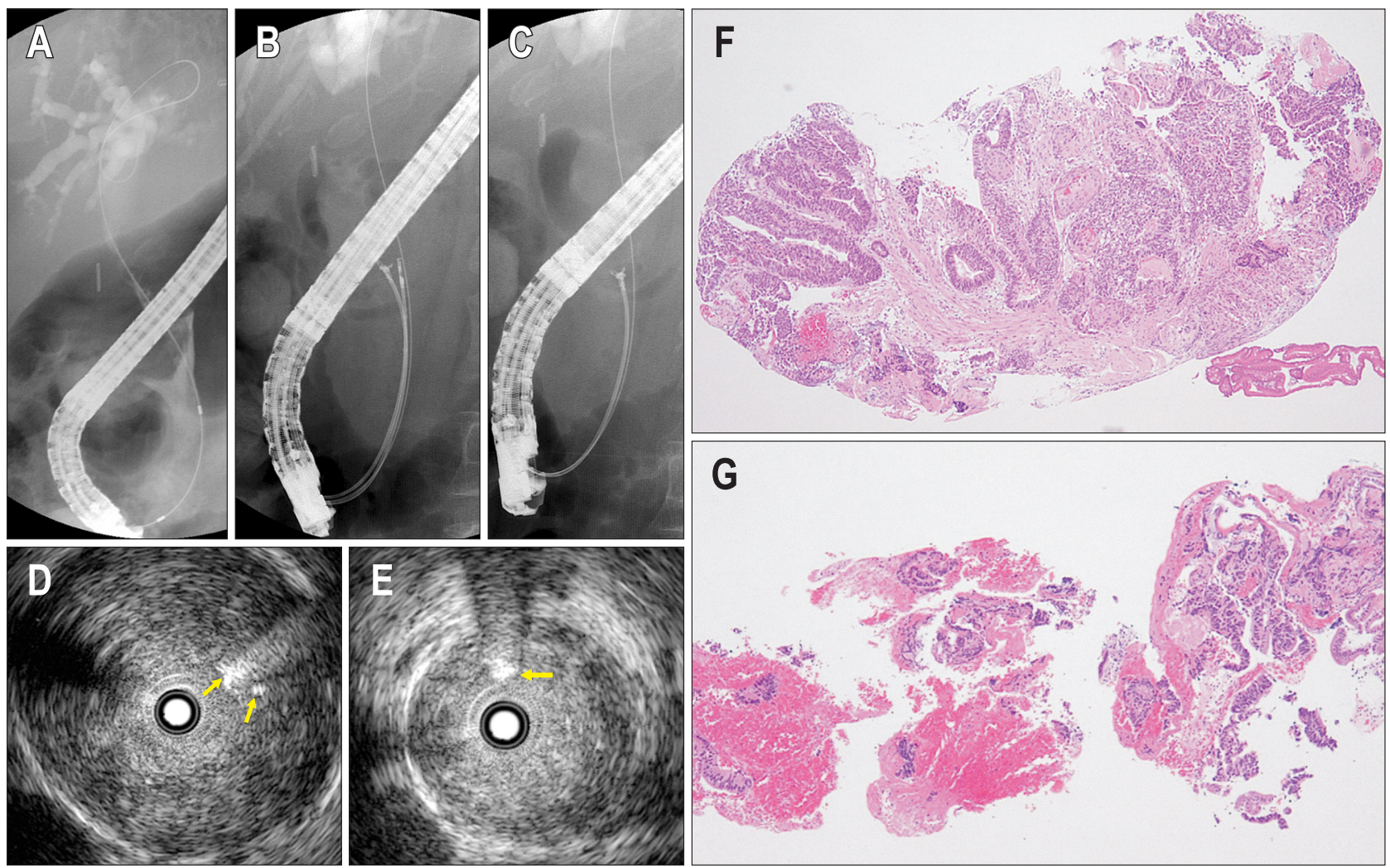

Fig. 2. Intraductal ultrasonography (IDUS)-guided transpapillary forceps biopsy (TPB) for malignant biliary stricture of the nodular type. (A) Cholangiogram showing a stricture caused by a luminal obstructing mass on the common bile duct. (B) IDUS-guided TPB and (C) conventional TPB were performed during endoscopic retrograde cholangiopancreatography. IDUS shows (D) an isoechoic nodular mass completely filling the lumen and hyperechoic substances indicating open biopsy forceps (arrows) and (E) closed biopsy forceps (arrow). Histologically, the tissue quality was better with $(\mathrm{F})$ IDUS-guided TPB $(\mathrm{H} \& \mathrm{E}, \times 100)$ than with $(\mathrm{G})$ conventional TPB $(\mathrm{H} \& \mathrm{E}, \times 100)$. Adenocarcinoma was diagnosed using IDUS-guided and conventional TPBs. 
sent. We excluded patients with at least one of the following: (1) biliary strictures due to underlying benign biliary diseases; (2) lesions invading the periampullary area; (3) history of previous biliary surgery except cholecystectomy; (4) bleeding tendency (international normalized ratio of $>1.5$ [0.85 to 1.25] or platelet count of $<80,000 / \mathrm{mm}^{3}\left[150,000\right.$ to $\left.\left.450,000 / \mathrm{mm}^{3}\right]\right)$; and (5) presence of any contraindication to ERCP. Written informed consent was obtained from all patients. This study was approved by the Institutional Review Board of Soonchunhyang University Hospital (IRB No. SCHBC 2013-02-007). This study was registered on the UMIN Clinical Trial Registry (UMIN 000020192).

\section{ERCP with IDUS}

All ERCP procedures were conducted by two experienced investigators using a standard duodenoscope (TJF-260 or 260V; Olympus Medical System Co., Ltd., Tokyo, Japan). Contrast (Omnipaque; GE Healthcare, Seoul, Korea) was injected to identify the location and length of the stricture. IDUS was performed during ERCP by the same endoscopist. A guidewire was introduced and advanced to the bile duct, after which a 2.0-mm- diameter ultrasonic probe with a frequency of $20 \mathrm{MHz}$ (UMG20-29R; Olympus Optical Co., Ltd., Tokyo, Japan) was inserted into the bile duct over the guidewire without prior endoscopic sphincterotomy (EST). The catheter generated high-resolution, real-time, cross-sectional images with an axial resolution of 0.1 $\mathrm{mm}$ and a maximum penetration of approximately $20 \mathrm{~mm}$. The IDUS images were prospectively reviewed by two experts. Based on morphology on IDUS, the biliary stricture was classified as one of the following: (1) nodular lesion, (2) intraductal infiltrating (sclerosing) lesion, or (3) extrinsic compressed lesion (Fig. 1).

\section{Conventional TPB}

A TPB was performed under fluoroscopic guidance using a clamshell-type needleless biopsy forceps (FB-39Q; Olympus Medical System) with a Teflon sheath (outer diameter of 1.8 $\mathrm{mm}$ ) after minor EST. A biopsy forceps was introduced into the bile duct and approached the distal part of the stricture with gentle pushing. Four biopsy specimens were obtained from each patient.
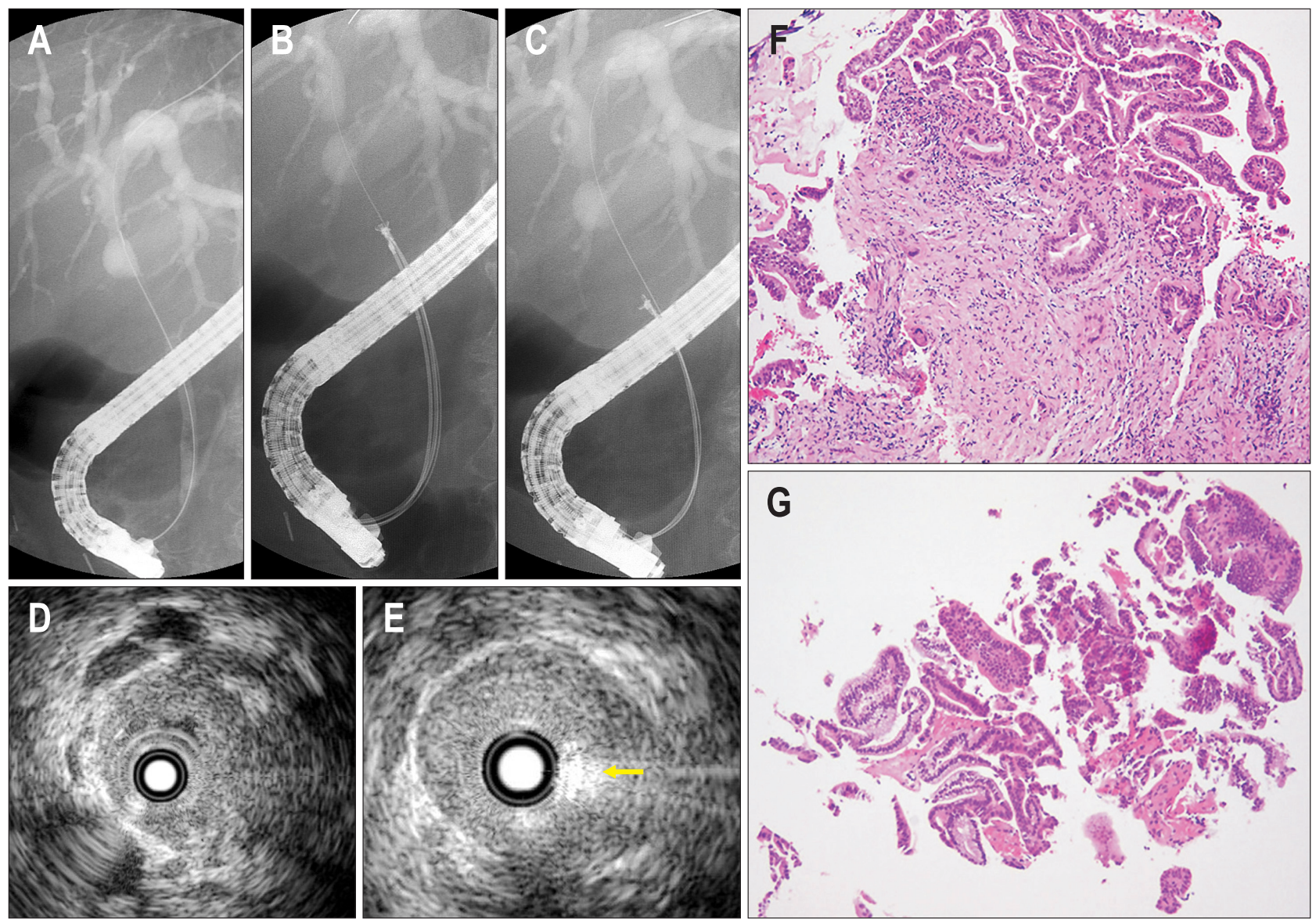

Fig. 3. Intraductal ultrasonography (IDUS)-guided transpapillary forceps biopsy (TPB) for malignant biliary strictures of the infiltrating type. (A) Cholangiogram showing a stricture on the common bile duct (CBD). (B) IDUS-guided TPB and (C) conventional TPB were performed during endoscopic retrograde cholangiopancreatography. IDUS shows (D) asymmetric, hypoechoic wall thickening on the CBD and (E) hyperechoic substances indicating closed biopsy forceps (arrow). Malignancy was only confirmed with (F) IDUS-guided TPB (H\&E, ×100) and not with (G) conventional TPB (H\&E, ×100). 


\section{IDUS-guided TPB}

An ultrasonic probe was inserted into the bile duct over the guidewire after EST, and IDUS revealed the segment of maximum bile duct wall thickening. To prevent pneumobilia, we performed minor EST and filled the bile duct with normal saline before IDUS. While maintaining the ultrasonic probe on the narrowest segment of stricture by the lesion, the same biopsy forceps for conventional biopsy was inserted into the orifice of the papilla to the tip of the placed ultrasonic probe by fluoroscopic guidance. TPB using biopsy forceps was then performed under ultrasonic visualization of the lesion and opening and closing of the biopsy forceps on the lesion. During and after the biopsy, the scanning ultrasonic probe remained in that position. As with conventional TPB, four specimens were obtained from each patient (Figs 2 and 3).

\section{Classification of biopsy results}

One expert pathologist blinded to the biopsy methods evaluated the pathological results of the biopsy specimens. The lesions were categorized as non-diagnostic, benign, atypical, and positive for malignancy. Samples that were considered positive were classified as positive for malignancy, whereas samples that were considered non-diagnostic, benign, and atypical were classified as negative for malignancy.

\section{Standard of reference for final diagnosis}

A finial diagnosis was made using various methods: (1) definite histologic proof of malignancy on surgical specimen or biopsy of metastatic lesion; (2) diagnosis of malignancy on TPB and compatible with malignant disease; and (3) no proof of malignancy on TPB and on clinical/imaging follow-up of at least 6 months.

\section{Study design}

The priority for performing IDUS-guided TPB or conventional TPB was assigned randomly at an allocation ratio of $1: 1$. Blocked randomization (block size 2) was performed by drawing sealed opaque envelopes. The primary outcome was the accuracy of conventional and IDUS-guided TPB in patients with suspected MBS. The secondary outcomes were the technical success rate of IDUS-guided TPB, the cancer detection rates of each biopsy method according to tumor morphology and location, and procedure-related adverse events. The diagnostic accuracy was defined as the ratio of the sum of true-positive and true-negative values divided by the number of lesions, where "true-positive" refers to the presence of malignant cells. Technical failure of IDUS-guided TPB was defined when the ultrasonic probe could not be passed through the strictured segment, or tissue sampling using biopsy forceps was impossible at the segment of maximum bile duct wall thickening as revealed by IDUS. The cancer detection rate was defined as the sum of true-positive results divided by the total number of malignant lesions. All patients underwent follow-up investigations with laboratory and radiological tests for at least 1 day after ERCP. Pancreatitis, bleeding at the site of biopsy, perforation, and cholangitis were recorded according to normal protocols.

\section{Statistical analysis}

Categorical parameters including sex and final diagnosis are expressed as frequencies and proportions. Continuous variables including age are reported as the median (range). Sensitivity and accuracy analysis was performed, and the data are reported with exact 95\% confidence intervals. The chi-square test and the Fisher exact test were applied for comparisons between the two groups. A p-value of less than 0.05 was considered to indicate a statistically significant difference between groups. All statistical data were analyzed using the SPSS for Windows software version 19.0 (IBM Corp., Armonk, NY, USA).

\section{RESULTS}

Between July 2012 and June 2014, 72 patients were enrolled; five patients were excluded for the following reasons: altered gastrointestinal anatomy $(n=2)$, failure of ERCP due to gastric outlet or duodenal obstruction $(\mathrm{n}=2)$, and contraindication for ERCP $(n=1)$. The remaining 67 patients were enrolled and underwent ERCP with IDUS. Among them, technical failure of IDUS-guided TPB was observed in two patients (3.0\%). Finally, a total of 65 patients (38 males; median age, 66.9 years [range, 48 to 85 years]) with suspected MBS were analyzed in this study. In 61 of 65 patients (93.8\%), MBS was diagnosed; the other four patients $(6.2 \%)$ were diagnosed with a benign biliary stricture. The tumor morphology according to IDUS findings was classified in 61 patients with MBS. The most common type was an intraductal infiltrating lesion in 29 patients (47.5\%). Nodular lesions and extrinsic compressed lesions were observed in 23 patients (37.7\%) and nine patients (14.8\%), respectively. The final diagnoses of MBS were bile duct cancer $(n=43)$, pancreatic can-

Table 1. Patient Characteristics $(n=65)$

\begin{tabular}{lc}
\hline \multicolumn{1}{c}{ Characteristic } & Value \\
\hline Age, median (range), yr & $66.9(48-85)$ \\
Male sex, $\mathrm{n}(\%)$ & $38(58.5)$ \\
Location of stricture, $\mathrm{n}(\%)$ & \\
$\quad$ Proximal & $21(32.3)$ \\
Mid & $27(41.5)$ \\
Distal & $17(26.2)$ \\
Final diagnosis, $\mathrm{n}(\%)$ & \\
Malignant & $61(93.8)$ \\
Benign & $4(6.2)$ \\
\hline
\end{tabular}




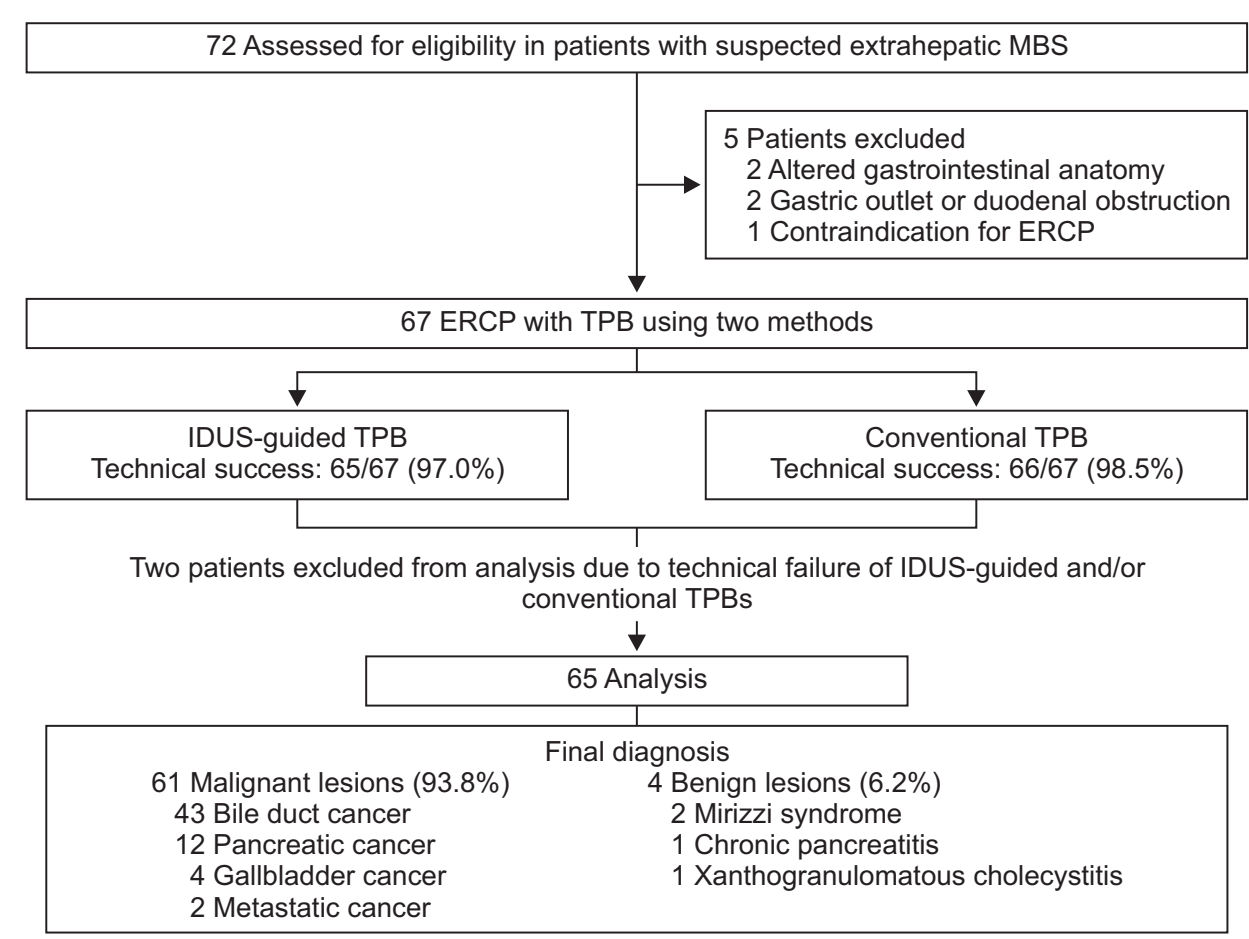

Fig. 4. Patient flow and final diagnosis.

MBS, malignant biliary stricture; ERCP, endoscopic retrograde cholangiopancreatography; TPB, transpapillary forceps biopsy; IDUS, intraductal ultrasonography.
Table 2. Pathological Results and Diagnostic Accuracy of TPBs in Patients with Suspected Malignant Biliary Stricture

\begin{tabular}{|c|c|c|c|}
\hline \multirow{2}{*}{ Pathological results } & \multicolumn{2}{|c|}{ Methods of TPB $(n=65)$} & \multirow{2}{*}{ p-value } \\
\hline & IDUS-guided TPB & Conventional TPB & \\
\hline Malignant & 55 & 46 & \\
\hline Atypia & 4 & 8 & \\
\hline Benign & 5 & 6 & \\
\hline Non-diagnostic & 1 & 5 & \\
\hline Accuracy, \% (95\% CI) & $90.8(83.7-97.8)$ & $76.9(66.7-87.5)$ & 0.027 \\
\hline
\end{tabular}

TPB, transpapillary forceps biopsy; IDUS, intraductal ultrasonography; CI, confidence interval.

Table 3. Comparison of Cancer Detection Rates Using TPBs According to the Tumor Morphology in Patients with Malignant Biliary Stricture

\begin{tabular}{lccc}
\hline \multirow{2}{*}{ Tumor morphology } & \multicolumn{2}{c}{ Positive for malignancy, $\mathrm{n}(\%)$} & \\
\cline { 2 - 3 } & $\begin{array}{c}\text { IDUS-guided } \\
\text { TPB }\end{array}$ & $\begin{array}{c}\text { Conventional } \\
\text { TPB }\end{array}$ & \\
\hline Nodular (n=23) & $22(95.7)$ & $21(91.3)$ & NS \\
Intraductal infiltration (n=29) & $26(89.6)$ & $19(65.5)$ & $0.028^{*}$ \\
Extrinsic compressed $(\mathrm{n}=9)$ & $7(77.8)$ & $6(66.7)$ & NS \\
Total (n=61) & $55(90.2)$ & $46(75.4)$ & $0.031^{*}$ \\
\hline
\end{tabular}

TPB, transpapillary forceps biopsy; IDUS, intraductal ultrasonography; NS, not significant.

${ }^{*}$ Chi-square test.
Table 4. Comparison of Cancer Detection Rates Using TPBs According to the Location of Strictures in Patients with Malignant Biliary Stricture

\begin{tabular}{lccc}
\hline \multirow{2}{*}{ Location of stricture } & \multicolumn{3}{c}{ Positive for malignancy, n (\%) } \\
\cline { 2 - 3 } & IDUS-guided TPB & Conventional TPB & \\
\hline Proximal $(\mathrm{n}=20)$ & $18(90.0)$ & $15(75.0)$ & NS \\
Mid $(\mathrm{n}=25)$ & $24(96.0)$ & $20(80.0)$ & NS \\
Distal $(\mathrm{n}=16)$ & $13(81.3)$ & $11(68.8)$ & NS \\
Total $(\mathrm{n}=61)$ & $55(90.2)$ & $46(75.4)$ & $0.031^{*}$ \\
\hline
\end{tabular}

TPB, transpapillary forceps biopsy; IDUS, intraductal ultrasonography; NS, not significant.

${ }^{*}$ Chi-square test.

cer $(n=12)$, gallbladder cancer $(n=4)$, and metastatic cancer $(n=2)$. The patients with a benign biliary stricture were diagnosed with Mirizzi syndrome $(n=2)$, chronic pancreatitis $(n=1)$, and xanthogranulomatous cholecystitis ( $\mathrm{n}=1$ ) (Table 1, Fig. 4).

The diagnostic accuracy of IDUS-guided TPB for MBS was significantly higher than that of conventional TPB $(90.8 \%$ vs 76.9\%, $\mathrm{p}=0.027$ ) (Table 2). Among 23 patients with nodular lesions, malignancies were confirmed in 22 patients (95.7\%) with IDUS-guided TPB and in 21 patients (91.3\%) with conventional TPB $(p=1.00)$. The cancer detection rate in the nine patients with extrinsic compressed lesions was not significantly different between IDUS-guided TPBs and conventional TPBs (77.8\% vs $66.7 \%, p=1.00$ ). However, among 29 cases with intraductal infiltrating lesions, IDUS-guided TPB showed a significantly higher cancer detection rate compared with conventional TPB (89.6\% vs 65.5\%, p=0.028) (Table 3). There was no significant 
difference in cancer detection rate according to the location of biliary stricture (Table 4).

There were no significant procedure-related adverse events except mild hemobilia in two patients (3.1\%) after TPB.

\section{DISCUSSION}

Endoscopic biopsy of suspected MBS lesions is highly desirable for adequate management. If a histological diagnosis is made at the first endoscopic procedure, further invasive diagnostic procedures can be avoided and appropriate treatment can be initiated promptly. ${ }^{2}$

The most widely used endoscopic procedure for tissue sampling in suspected MBS is ERCP-based sampling methods including brush cytology and TPB. ${ }^{7}$ However, the sensitivities of brush cytology and TPB for diagnosing MBS remain unsatisfactory. ${ }^{1,7-9}$ A meta-analysis of the effectiveness of brush cytology and TPB showed sensitivities of $45.0 \%$ and $48.1 \%$, respectively. Combining the two methods showed a sensitivity of $59.4 \% .{ }^{1}$ These insufficient results were likely caused by the fact that tissue sampling during TPB is performed blindly under fluoroscopic guidance. In addition, MBS lesions that infiltrate the duct or manifest as extrinsic compressing masses are especially difficult to diagnose pathologically using ERCP-based tissue sampling.

To overcome the limitations of ERCP-based sampling, several diagnostic modalities have been studied, including cholangioscopy-guided tissue sampling and endoscopic ultrasound-guided fine needle aspiration biopsy (EUS-FNAB). Peroral cholangioscopy permits direct visualization of the biliary tree with targeted tissue sampling. The sensitivity of peroral cholangioscopy-guided tissue sampling has been reported to range from 57.0\% to $76.5 \% .{ }^{10-12}$ Draganov et al. ${ }^{11}$ evaluated the accuracy of SpyGlass peroral cholangioscopy-guided mini-forceps biopsy (Boston Scientific, Natick, MA, USA) and compared it with ERCP-based sampling in patients with indeterminate bile duct strictures. In that study, the accuracy was $84.6 \%$ for SpyGlass peroral cholangioscopy-guided biopsy, which also showed significantly better sensitivity. However, SpyGlass peroral cholangioscopy has some limitations to its use such as technical difficulty and high cost. ${ }^{13}$

EUS-FNAB is currently the standard procedure for tissue biopsy of solid pancreatic mass lesions with its high diagnostic yield. In previous meta-analyses, the sensitivity of EUS-FNAB has ranged from $85 \%$ to $89 \%$ for solid pancreatic lesions. ${ }^{14-16}$ However, EUS-FNAB has some limitations in cases of MBS other than pancreatic mass lesions. ${ }^{17,18}$

IDUS with high-frequency probes is an imaging modality used to diagnose pancreatobiliary disease. These probes can provide a $360^{\circ}$ sector scan, and can be easily introduced into the bile duct without performing an EST and advanced through a stricture. IDUS can provide real-time, high-resolution images of the entire bile duct wall and periductal tissue. IDUS can also be useful to distinguish malignant from benign biliary strictures and showed higher diagnostic accuracy when compared with EUS. ${ }^{4-6,19}$ In previous studies, the sensitivity of IDUS for the diagnosis of MBS has ranged from $83 \%$ to $89 \% .^{20,21}$ Features evident on IDUS that suggest malignancy include disruption of the normal bile duct structure, hypoechoic masses with irregular margins, heterogeneous echo-poor areas invading surrounding tissue, and continuation of the main hypoechoic mass into adjacent structures. Also, IDUS can be useful for local T staging of tumors. ${ }^{4,5,19}$ Therefore, it seems that the sensitivity of TPB can be improved if biopsy is performed under IDUS because this allows more accurate targeting of suspicious lesions through visualizing the mass, wall layer, and biopsy forceps when compared with TPB under fluoroscopic guidance.

Tamada et $a l^{22}$ classified the IDUS images of these tumors as polypoid lesions, localized wall thickening, intraductal sessile tumors, sessile tumors outside of the bile duct, or absence of an apparent lesion, and compared IDUS images with the biopsy results. In this study, when the IDUS images showed a polypoid lesion and intraductal sessile tumor, bile duct biopsy showed sensitivities of $80 \%$ and $92 \%$, respectively. However, when the IDUS images showed a sessile tumor outside of the bile duct, biopsy showed a relatively low sensitivity of 50\%. Based on the results of this study, it appears that ERCP-based TPB has limited utility for biopsies in patients with extrinsic mass lesions.

We performed TPB during IDUS for more accurate biopsy results and evaluated the improvement in sensitivity of IDUSguided TPBs with classification of the MBS as a nodular lesion, intraductal infiltrating lesion, or extrinsic compressed lesion. In the current study, an intraductal infiltrating lesion was the most common finding on IDUS (47.5\%). IDUS-guided TPB showed a significant improvement of the diagnostic accuracy in patients with MBSs, especially in cases with intraductal infiltrating lesions. Unlike nodular lesions, in cases with intraductal infiltrating lesions, it is difficult to accurately identify the lesion to be biopsied using only fluoroscopy, because this type of lesion is not obvious on this modality. However, with IDUS, endoscopists can accurately target the bile duct lesion and can perform a biopsy using forceps in real-time, often improving the sensitivity of the biopsy results. Unfortunately, our study did not show significant improvement of cancer detection rates in cases with extrinsic compressed lesions.

Our study had several limitations. First, since there are no accessories that combine IDUS and forceps biopsy, IDUS-guided TPB is more complicated than conventional TPB. Second, there is a possibility of mechanical trauma with the IDUS probe during TPB with biopsy forceps. Third, our sample size was relatively small, so further studies should be performed with larger numbers of patients to verify our results. Further studies should directly compare the sensitivity of IDUS-guided TPB with other 
tissue sampling methods, such as EUS-FNAB and peroral cholangioscopy-guided bile duct biopsy. Fourth is the short followup period of 6 months. However, all benign lesions were followed for more than 12 months. Fifth, the cost-effectiveness of the IDUS-guided TPB in the diagnosis of biliary stricture could not be determined in this study.

In conclusion, significantly higher diagnostic yields can be obtained with IDUS-guided TPB compared with conventional TPB in patients with suspected MSBs. It is expected that a new type of IDUS probe or accessories for IDUS-guided TPB will allow more accurate targeting of lesions during biopsy and will further improve cancer detection rates.

\section{CONFLICTS OF INTEREST}

No potential conflict of interest relevant to this article was reported.

\section{ACKNOWLEDGEMENTS}

This work was supported in part by the Soonchunhyang University Research Fund.

We thank A Ri Song, RN; Song Ah Jeong, RN; Seon Young Moon, RN; and the rest of the nursing staff for their assistance with the procedures.

\section{REFERENCES}

1. Navaneethan U, Njei B, Lourdusamy V, Konjeti R, Vargo JJ, Parsi MA. Comparative effectiveness of biliary brush cytology and intraductal biopsy for detection of malignant biliary strictures: a systematic review and meta-analysis. Gastrointest Endosc 2015;81:168-176.

2. De Bellis M, Sherman S, Fogel EL, et al. Tissue sampling at ERCP in suspected malignant biliary strictures (Part 1). Gastrointest Endosc 2002;56:552-561.

3. de Bellis M, Sherman S, Fogel EL, et al. Tissue sampling at ERCP in suspected malignant biliary strictures (Part 2). Gastrointest Endosc 2002;56:720-730.

4. Vazquez-Sequeiros E, Baron TH, Clain JE, et al. Evaluation of indeterminate bile duct strictures by intraductal US. Gastrointest Endosc 2002;56:372-379.

5. Domagk D, Wessling J, Reimer P, et al. Endoscopic retrograde cholangiopancreatography, intraductal ultrasonography, and magnetic resonance cholangiopancreatography in bile duct strictures: a prospective comparison of imaging diagnostics with histopathological correlation. Am J Gastroenterol 2004;99:1684-1689.

6. Kim HS, Moon JH, Choi HJ, et al. The role of intraductal US in the management of idiopathic recurrent pancreatitis without a definite cause on ERCP. Gastrointest Endosc 2011;73:1148-1154.

7. Weilert F, Bhat YM, Binmoeller KF, et al. EUS-FNA is superior to
ERCP-based tissue sampling in suspected malignant biliary obstruction: results of a prospective, single-blind, comparative study. Gastrointest Endosc 2014;80:97-104.

8. Rösch T, Hofrichter K, Frimberger E, et al. ERCP or EUS for tissue diagnosis of biliary strictures? A prospective comparative study. Gastrointest Endosc 2004;60:390-396.

9. Jailwala J, Fogel EL, Sherman S, et al. Triple-tissue sampling at ERCP in malignant biliary obstruction. Gastrointest Endosc 2000;51(4 Pt 1):383-390.

10. Hartman DJ, Slivka A, Giusto DA, Krasinskas AM. Tissue yield and diagnostic efficacy of fluoroscopic and cholangioscopic techniques to assess indeterminate biliary strictures. Clin Gastroenterol Hepatol 2012;10:1042-1046.

11. Draganov PV, Chauhan S, Wagh MS, et al. Diagnostic accuracy of conventional and cholangioscopy-guided sampling of indeterminate biliary lesions at the time of ERCP: a prospective, long-term follow-up study. Gastrointest Endosc 2012;75:347-353.

12. Chen YK, Pleskow DK. SpyGlass single-operator peroral cholangiopancreatoscopy system for the diagnosis and therapy of bileduct disorders: a clinical feasibility study (with video). Gastrointest Endosc 2007;65:832-841.

13. Sun X, Zhou Z, Tian J, et al. Is single-operator peroral cholangioscopy a useful tool for the diagnosis of indeterminate biliary lesion? A systematic review and meta-analysis. Gastrointest Endosc 2015;82:79-87.

14. Wani S, Muthusamy VR, Komanduri S. EUS-guided tissue acquisition: an evidence-based approach (with videos). Gastrointest Endosc 2014;80:939-959.

15. Hewitt MJ, McPhail MJ, Possamai L, Dhar A, Vlavianos P, Monahan KJ. EUS-guided FNA for diagnosis of solid pancreatic neoplasms: a meta-analysis. Gastrointest Endosc 2012;75:319-331.

16. Lee YN, Moon JH, Kim HK, et al. Core biopsy needle versus standard aspiration needle for endoscopic ultrasound-guided sampling of solid pancreatic masses: a randomized parallel-group study. Endoscopy 2014;46:1056-1062.

17. DeWitt J, Misra VL, Leblanc JK, McHenry L, Sherman S. EUSguided FNA of proximal biliary strictures after negative ERCP brush cytology results. Gastrointest Endosc 2006;64:325-333.

18. Mohamadnejad M, DeWitt JM, Sherman S, et al. Role of EUS for preoperative evaluation of cholangiocarcinoma: a large singlecenter experience. Gastrointest Endosc 2011;73:71-78.

19. Farrell RJ, Agarwal B, Brandwein SL, Underhill J, Chuttani R, Pleskow DK. Intraductal US is a useful adjunct to ERCP for distinguishing malignant from benign biliary strictures. Gastrointest Endosc 2002;56:681-687.

20. Stavropoulos S, Larghi A, Verna E, Battezzati P, Stevens P. Intraductal ultrasound for the evaluation of patients with biliary strictures and no abdominal mass on computed tomography. Endoscopy 2005;37:715-721.

21. Tamada K, Ueno N, Tomiyama T, et al. Characterization of biliary strictures using Intraductal ultrasonography: comparison 
with percutaneous cholangioscopic biopsy. Gastrointest Endosc 1998;47:341-349.

22. Tamada K, Tomiyama T, Wada S, et al. Endoscopic transpapillary bile duct biopsy with the combination of Intraductal ultrasonography in the diagnosis of biliary strictures. Gut 2002;50:326-331. 